\title{
Potential resident bacterial microbiota in udder tissues of culled cows sampled in abattoir
}

\author{
Barbara Pirard $^{\mathrm{a}, *}$, Sébastien Crèvecoeur ${ }^{\mathrm{a}}$, Papa Abdoulaye Fall ${ }^{\mathrm{b}}$, Patrick Lausberg ${ }^{\mathrm{c}}$, \\ Bernard Taminiau ${ }^{a}$, Georges Daube ${ }^{\mathrm{a}}$ \\ ${ }^{a}$ Fundamental and Applied Research for Animals \& Health (FARAH), Faculté de Médecine Vétérinaire, Département des Sciences des Denrées Alimentaires, Université de \\ Liège, Avenue de Cureghem 10, 4000 Liège, Belgium \\ ${ }^{\mathrm{b}}$ Genalyse Partner SA, Rue Hayeneux 62, 4040 Herstal, Belgium \\ ${ }^{\mathrm{c}}$ Pegri, Alte Aachener Strasse 15, 4780 Saint Vith, Belgium
}

\section{A R T I C L E I N F O}

\section{Keywords:}

Udder health

16SrDNA amplicon sequencing

Bovine milk

Bovine mammary gland

Bacterial microbiota

\begin{abstract}
A B S T R A C T
While mammary gland tissues (MGTs) are difficult to sample without risks for cow's health or milk production, milk analysis are used in routine to assess dairy cow udder's health. This study aimed to identify, quantify, compare the milk and MGTs microbiota of macroscopically healthy dairy bovine mammary glands (MG) in order to evaluate their degree of similarity. We harvested 13 couples of milk and MGTs samples, originated from the same quarter at culling. 16S rDNA Amplicon Sequencing was performed, showing Corynebacterium as the main bacterial genus in both types of samples but generally found in the milk in higher proportions than in tissues. Species evenness was higher in MGTs while species richness was higher in milk samples. Beta diversity was significantly different between both matrices suggesting the presence of a resident microbiota in MGTs of dairy cows at time of culling partially reflected by the milk microbiota from the same quarter.
\end{abstract}

Aseptic milking samples and microbiological analyses are used as gold standard for bovine mastitis diagnosis (Oikonomou et al., 2012). Several recent studies based on 16S rDNA amplicon sequencing, revealed a complex microbiota in mastitic milk (Ganda et al., 2016) as in healthy quarters (Bonsaglia et al., 2017) and some authors go so far as to claim that bovine MG possesses its own microbiome (Ganda et al., 2017; Oikonomou et al., 2014), what's controversial by others (Rainard, 2017). Indeed, emergence of the concept of intramammary microbiota contradicts the traditional view that milk in a healthy udder quarter is sterile (Derakhshani et al., 2018; Rainard, 2017).

Therefore, this preliminary study aimed to test the relevance of milk microbiota analysis in order to evaluate the MG microbiota. For this purpose, milk and MGTs samples of macroscopically healthy dairy bovine MG have been taken in abattoir and analysed to identify, quantify, compare the milk and MGTs microbiota, by amplicon sequencing of 16S rDNA V1-V3 regions.

Thirteen couples of milk and MGTs from the same quarter of 7 reformed cows (one to two quarter per cow; for more information on sampled animals see Appendix D: Supplementary materials), were sampled in a Belgian slaughterhouse in March 2018. Sampled cows presented all macroscopically healthy MG at clinical exam. Aseptic milking has been done just before culling, following procedure reported by Lima et al. (2017). The teats were first disinfected with iodized soap (Isobetadine ${ }^{\circledR}$ ), carefully cleaned using disposable absorbent paper, and finally scrubbed with $70 \%$ alcohol. After ejection of the five first jets, milking was realized in sterile plastic tubes, wearing disposable gloves. MGTs had been taken off the carcasses on slaughter line. After the chain operator had excised the lower part of the udder (teats and gland cisterns), the healthy macroscopical aspect of the parenchyma was checked and a cubic decimeter of tissues was cut from the previously milked mammary quarters. A smaller cube of one cubic centimeter was then excised with a sterile single-use scalpel from the center of the first tissue bloc. The incision site of this first bloc has been cauterized with a flameheated metal, before excision of the second cube, in order to avoid bacterial translocation from the surface to the center. This second cube has finally been suspended in sterile physiological water and then vortexed $(1400 \mathrm{rpm})$ to obtain bacterial suspensions.

Total DNA was extracted with DNeasy Blood \& Tissue kit (Qiagen) and a negative control with nuclease free-water were included. Metagenetic and data analysis were performed as described previously,

\footnotetext{
* Corresponding author.

E-mail address: barbara.pirard@uliege.be (B. Pirard).
} 


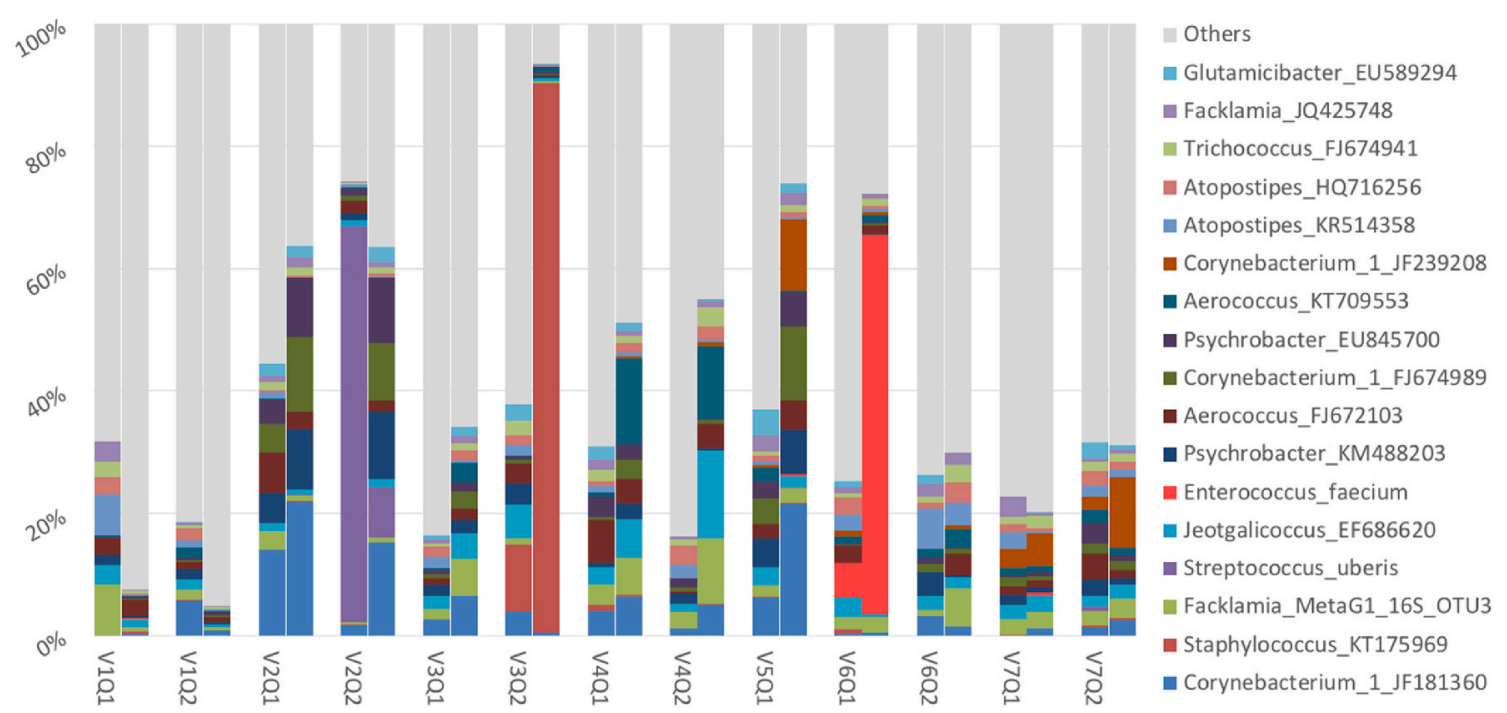

Fig. 1. Taxa with a mean relative abundance upper $1 \%$ regarding to all samples represented pair by pair (MGTs and milk) and quarter by quarter. The bar-plot on the left of each pair represents mammary gland tissue of the quarter, the bar-plot on the right represents the milk.

Three quarters (V2Q2; V3Q2; V6Q1) show pathogenic bacteria at a high level (Streptococcus uberis, Staphylococcus_KT175069, Enterococcus faecium respectively). Abbreviations: V, cow; Q, quarter.

amplifying V1-V3 regions from the $16 \mathrm{~S}$ rDNA and then using Illumina MiSeq ${ }^{\circledR}$ technology (Illumina, SY-410-1003) (Ngo et al., 2018). Sequencing reads processing was realized like done before, with Mothur (v1.39.5) (https://www.mothur.org) for alignment and clustering, and UCHIME algorithm (https://www.drive5.com/uchime/) for chimera detection (Edgar et al., 2011; Rodriguez et al., 2016). A distance of 0,03 was used for OTU generation. $16 \mathrm{~S}$ reference alignment and taxonomical assignment was based upon SILVA database (v.128) (https://www.arb -silva.de) of full-length 16S rDNA sequences (Ngo et al., 2018; Quast et al., 2013).

Alpha diversity was assessed by the Reciprocal Simpson diversity index, joining Chao1 index and Simpson evenness, realizing a paired non-parametric Wilcoxon test. Beta diversity was evaluated analysing molecular variance (AMOVA) and homogeneity of molecular variance (HOMOVA) using Bray Curtis index. LEfSe program (Huttenhower lab) was used in order to test specific biomarkers in both matrices.

A total of 4683 operational taxonomic units (OTUs) at the level of 99\% of similarities according to SILVA database (v.128) was found. Pathogenic bacteria were dominant in three couples (Fig. 1). When detected in milk samples, the same pathogen was detected in MGTs from the same quarter: Streptococcus uberis (MGTs: 65\%; milk: 8\%); Staphylococcus spp. (MGTs: 11\%; milk: 90\%); Enterococcus faecium (MGTs: 6\%; milk: 62\%) (Fig. 1). Theses samples were removed for statistical analysis to eliminate bias.

Among the most represented taxa (mean relative abundance (MRA) $>1 \%$ ) in MGTs, the five most abundant phyla were Firmicutes, Actinobacteria, Bacteroidetes, Proteobacteria, Tenericutes. They were present in milk too, although only Firmicutes, Actinobacteria and Bacteroidetes are part of milk taxa with a MRA $>1 \%$. In the MGTs and milk, 9 and 10 species with a MRA $>1 \%$ were respectively identified in all samples (see Appendices A and B: Supplementary materials).

The most abundant genus in both matrices was Corynebacterium, found in all samples, generally in higher proportions in the milk (Fig. 1). Among it, the most important specie, Corynebacterium_FJ674989, found in all samples too $(2,81 \pm 1,63 \%$ in MGTs; $3,47 \pm 4,70 \%$ in milk), was very close to $C$. fecaleae $\left(98,36 \%\right.$ similarities in Blast ${ }^{\circledR}$ NCBI data bank). In tissues, Flavobacterium_FJ820431 (LDA:3,69; P:0,0011) and Atopostipes_KR514358 (LDA:3,90; P:0,00169647) taxa were statistically more abundant than in milk.

Simpson evenness was higher in MGTs than in milk, but richness (Chao index) was higher in milk samples $(P<0,5)$ (see Appendix C:

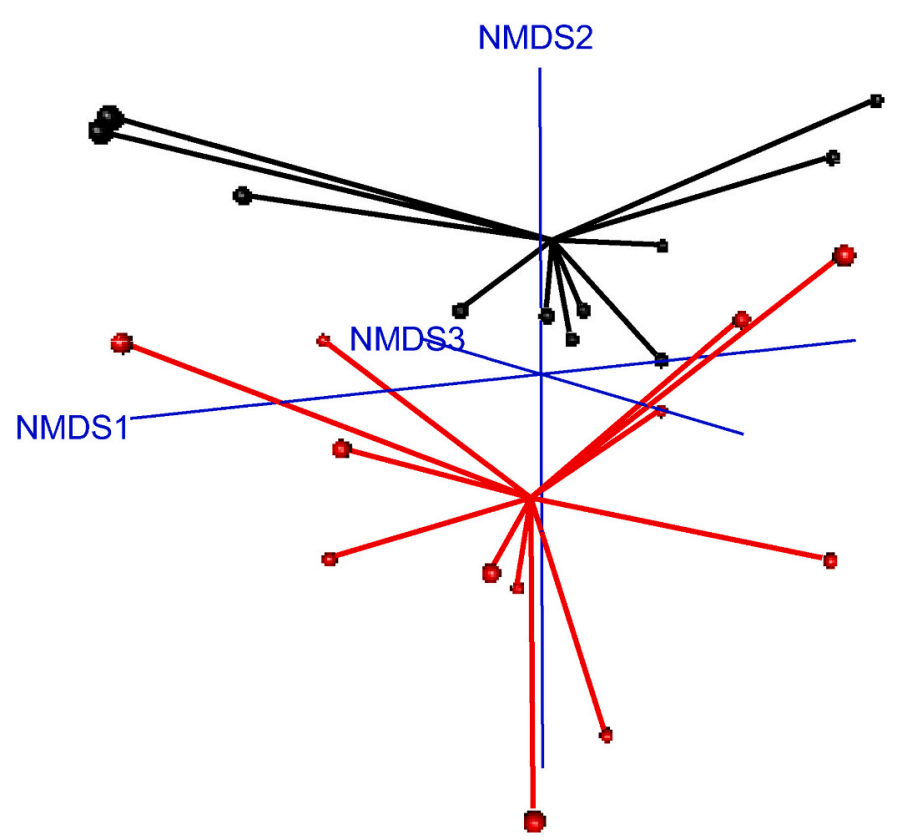

Fig. 2. Non-parametric multidimensional scaling (NMDS) representation of milk (black balls) and mammary gland tissue (red balls) samples based on a Bray-Curtis dissimilarity matrix. The accepted model presents 4 dimensions $(\mathrm{k})$ for an acceptable stress value of $0,0760(<0,1)$. The centromere of the two groups (equidistant point between samples of the same group) is illustrated by a "spider web" connecting the samples. (For interpretation of the references to colour in this figure legend, the reader is referred to the web version of this article.)

Supplementary materials). AMOVA and HOMOVA analysis, testing Bray Curtis dissimilarity index, revealed a significative difference of the beta diversity between MGTs and milk (Fig. 2). AMOVA analysis $(P<0,5)$, shown a significantly different clustering of MGTs from milk. While HOMOVA analysis $(P<0,5)$, shown a significative different dispersion between the samples of both matrices. That implies that milk and MGTs present two distinct populations in composition and structure. No significative clustering of bacterial populations from samples of a same 
animal were found (results not shown).

The most identified taxa in all samples, are not equally distributed between MGTs and milk suggesting that some organisms could be favoured in each matrix. However, as shown by Fig. 1, almost all milk major taxa are also predominant in MGTs (see also Appendices A and B: Supplementary materials). This redundancy suggests that both microbiota are well similar in nature, albeit distinct if we refer to the results of the AMOVA and HOMOVA tests (Fig. 2). Therefore, from an ecological perspective, this may correspond two different ecological niches forming part of a complex ecosystem as it has been described for mammalian body (Costello et al., 2012).

The five most abundant phyla found in MGTs (Firmicutes, Actinobacteria, Bacteroidetes, Proteobacteria and Tenericutes) confirm what has already been reported in recent studies describing healthy and mastitic colostrum from primiparous and multiparous cows (Lima et al., 2017; Yeoman et al., 2018). Also, several families and genera we found in both matrices have been previously described in recent studies about individual healthy milk quarters such as Staphylococcaceae, Corynebacteriaceae, Aerococaceae, Moraxellaceae (Ganda et al., 2016, 2017) and Corynebacterium or Psychrobacter (Kuehn et al., 2013). Intriguingly we identified Clostridiaceae and Lachnospiraceae in both matrices, but only in MGT with a MRA > $1 \%$ (see Appendices A and B: Supplementary materials). These two gut-associated families, were identified as predominant in the teat canal (TC) microbiota (Gill et al., 2006). This reinforces the concept of a possible influence of the TC microbiota on the MGT's microbial colonization detailed in the review work of Derakhshani et al. (2018).

The genus Corynebacterium is a facultative anaerobic common host commensal and environmental bacterium that has been detected like one of the most abundant genera of the udder skin (Yeoman et al., 2018). Atopostipes, pointed by LEfSe test as one of the most abundant taxa in MGT, is a Gram positive facultative anaerobic lactic-acid-bacteria (Lawson and Caldwell, 2014). Interestingly, it has been established in the literature that innate immune system of ruminants adapts the response specifically regarding to some Gram-positive bacteria (Rainard and Riollet, 2006), whose represent principal part of major taxa in our results (see Appendices A and B: Supplementary materials). Moreover, we also know that some lactic acid bacteria originating from the milk and the TC can adhere to and internalize bovine MGTs' epithelial cells (Bouchard et al., 2015).

Although these results and observations do not constitute irrefutable evidence of the existence of a residential microbiota inside MGT of dairy cows, this work offer an insight on the exploration of this hypothesis. The bovine MG has been considered for long as a sterile environment. This concept was based on routine clinical culture assays applied to aseptically taken milk samples (Neave, 1975; Rainard, 2017). However, it has been reported after that culture negative milk samples, sterilely milked from healthy and mastitic quarters contain a large microbial communities' panel (Kuehn et al., 2013; Vasquez et al., 2019). Several explanations may exist, including inability to culture all the bacteria present (Kuehn et al., 2013), methodological differences between novel DNA sequencing techniques and conventional culture-based approach (Derakhshani et al., 2018), but also contamination that can occur during any step of sample handling and analysis, via laboratory reagents like in DNA extraction kits in some cases (Salter et al., 2014; Taponen et al., 2019).

To our knowledge, this is the first study reporting microbiological comparison between MGTs and milk, in order to assess their correlation in the context of bovine MG's health. We identified here microbial communities in milk and MGTs that are different in structure, but partially similar in terms of composition. These results suggest that, if there is a microbiota in MGTs of dairy cows at time of culling, it can partially be reflected by the milk microbiota from the same quarter. This corroborate conclusions of many papers, suggesting that amplicon sequencing of the $16 \mathrm{~S}$ rDNA from milk samples should be a great diagnostic tool to develop to evaluate MG's health of dairy cows (Ganda et al., 2016; Kuehn et al., 2013; Oikonomou et al., 2014).

However, in order to better explore hypothesis of the residential character of a microbiota inside MGT of dairy cows, a larger number of samples needs to be tested, taking in account and exploring individual parameters of animals like suggested recently (Derakhshani et al., 2018).

\section{Declaration of Competing Interest}

None of the authors of this paper has a financial or personal relationship with other people or organisations that could inappropriately influence or bias the content of the paper.

\section{Acknowledgements}

We thank the slaughterhouse of Saint-Vith and its kind personnel for their collaboration, as well as the students who provided assistance to facilitate the sampling.

Preliminary results were presented as an Abstract at the MBIO Colloquium of Adebiotech, Paris, 19-20 June 2018.

This research did not receive any specific grant from funding agencies in the public, commercial, or not-for-profit sectors.

\section{Supplementary data}

Supplementary data to this article can be found online at https://doi. org/10.1016/j.rvsc.2021.03.012.

\section{References}

Bonsaglia, E.C.R., Gomes, M.S., Canisso, I.F., Zhou, Z., Lima, S.F., Rall, V.L.M., Oikonomou, G., Bicalho, R.C., Lima, F.S., 2017. Milk microbiome and bacterial load following dry cow therapy without antibiotics in dairy cows with healthy mammary gland. Sci. Rep. 7, 8067.

Bouchard, D.S., Seridan, B., Saraoui, T., Rault, L., Germon, P., Gonzalez-Moreno, C., Nader-Macias, F.M., Baud, D., François, P., Chuat, V., 2015. Lactic acid bacteria isolated from bovine mammary microbiota: potential allies against bovine mastitis. PLoS One 10, e0144831.

Costello, E.K., Stagaman, K., Dethlefsen, L., Bohannan, B.J.M., Relman, D.A., 2012. The application of ecological theory toward an understanding of the human microbiome. Science 336, 1255-1262.

Derakhshani, H., Fehr, K.B., Sepehri, S., Francoz, D., De Buck, J., Barkema, H.W., Plaizier, J.C., Khafipour, E., 2018. Invited review: microbiota of the bovine udder: contributing factors and potential implications for udder health and mastitis susceptibility. J. Dairy Sci. 101, 10605-10625.

Edgar, R.C., Haas, B.J., Clemente, J.C., Quince, C., Knight, R., 2011. UCHIME improves sensitivity and speed of chimera detection. Bioinformatics 27, 2194-2200.

Ganda, E.K., Bisinotto, R.S., Lima, S.F., Kronauer, K., Decter, D.H., Oikonomou, G., Schukken, Y.H., Bicalho, R.C., 2016. Longitudinal metagenomic profiling of bovine milk to assess the impact of intramammary treatment using a third-generation cephalosporin. Sci. Rep. 6, 37565.

Ganda, E.K., Gaeta, N., Sipka, A., Pomeroy, B., Oikonomou, G., Schukken, Y.H., Bicalho, R.C., 2017. Normal milk microbiome is reestablished following experimental infection with Escherichia coli independent of intramammary antibiotic treatment with a third-generation cephalosporin in bovines. Microbiome 5, 74.

Gill, J.J., Sabour, P.M., Gong, J., Yu, H., Leslie, K.E., Griffiths, M.W., 2006. Characterization of bacterial populations recovered from the teat canals of lactating dairy and beef cattle by 16S rRNA gene sequence analysis. FEMS Microbiol. Ecol. 56, $471-481$.

Kuehn, J.S., Gorden, P.J., Munro, D., Rong, R., Dong, Q., Plummer, P.J., Wang, C., Phillips, G.J., 2013. Bacterial community profiling of milk samples as a means to understand culture-negative bovine clinical mastitis. PLoS One 8 (4), e61959.

Lawson, P.A., Caldwell, M.E., 2014. The family carnobacteriaceae. In: Rosenberg, E., DeLong, E.F., Lory, S., Stackebrandt, E., Thompson, F. (Eds.), The Prokaryotes: Firmicutes and Tenericutes. Springer, Berlin, Heidelberg, pp. 19-65.

Lima, S.F., Teixeira, A.G.V., Lima, F.S., Ganda, E.K., Higgins, C.H., Oikonomou, G., Bicalho, R.C., 2017. The bovine colostrum microbiome and its association with clinical mastitis. J. Dairy Sci. 100, 3031-3042.

Neave, F.K., 1975. Diagnosis of mastitis by bacteriological methods alone (v.85). In: Proceedings of the IDF Seminar on Mastitis Control 1975. Reading University, pp. 19-36. April 7-11.

Ngo, J., Taminiau, B., Fall, P.A., Daube, G., Fontaine, J., 2018. Ear canal microbiota - a comparison between healthy dogs and atopic dogs without clinical signs of otitis externa. Vet. Dermatol. 29, 425-e140.

Oikonomou, G., Machado, V.S., Santisteban, C., Schukken, Y.H., Bicalho, R.C., 2012. Microbial diversity of bovine Mastitic Milk as described by pyrosequencing of metagenomic 16s rDNA. PLoS One 7 (10), e47671. 
Oikonomou, G., Bicalho, M.L., Meira, E., Rossi, R.E., Foditsch, C., Machado, V.S., Teixeira, A.G.V., Santisteban, C., Schukken, Y.H., Bicalho, R.C., 2014. Microbiota of Cow's Milk; distinguishing healthy, sub-clinically and clinically diseased quarters. PLoS One 9 (1), e85904.

Quast, C., Pruesse, E., Yilmaz, P., Gerken, J., Schweer, T., Yarza, P., Peplies, J., Glöckner, F.O., 2013. The SILVA ribosomal RNA gene database project: improved data processing and web-based tools. Nucleic Acids Res. 41, D590-D596.

Rainard, P., 2017. Mammary microbiota of dairy ruminants: fact or fiction? Vet. Res. 48. Rainard, P., Riollet, C., 2006. Innate immunity of the bovine mammary gland. Vet. Res. 37, 369-400

Rodriguez, C., Taminiau, B., Korsak, N., Avesani, V., Van Broeck, J., Brach, P., Delmée, M., Daube, G., 2016. Longitudinal survey of Clostridium difficile presence and gut microbiota composition in a Belgian nursing home. BMC Microbiol. 16, 229.
Salter, S.J., Cox, M.J., Turek, E.M., Calus, S.T., Cookson, W.O., Moffatt, M.F., Turner, P., Parkhill, J., Loman, N.J., Walker, A.W., 2014. Reagent and laboratory contamination can critically impact sequence-based microbiome analyses. BMC Biol. 12, 87.

Taponen, S., McGuinness, D., Hiitiö, H., Simojoki, H., Zadoks, R., Pyörälä, S., 2019.

Bovine milk microbiome: a more complex issue than expected. Vet. Res. Paris 50 (44).

Vasquez, A.k., Ganda, E.k., Capel, M.b., Eicker, S., Virkler, P.d., Bicalho, R.c., Nydam, D. v., 2019. The microbiome of Escherichia coli and culture-negative nonsevere clinical mastitis: characterization and associations with linear score and milk production. J. Dairy Sci. 102, 578-594.

Yeoman, C.J., Ishaq, S.L., Bichi, E., Olivo, S.K., Lowe, J., Aldridge, B.M., 2018. Biogeographical differences in the influence of maternal microbial sources on the early successional development of the bovine neonatal gastrointestinal tract. Sci. Rep. 8, 3197. 